\title{
Magnetization Dynamics due to Pure Spin Currents in Magnetic Double Layers
}

\author{
G. Woltersdorf, ${ }^{1}$ O. Mosendz, ${ }^{2}$ B. Heinrich, ${ }^{2}$ and C. H. Back ${ }^{1}$ \\ ${ }^{1}$ Universität Regensburg, Universitätsstraße 31, 93040 Regensburg, Germany \\ ${ }^{2}$ Simon Fraser University, 8888 University Drive, V5A 1S6 Burnaby BC, Canada
}

(Received 22 June 2007; published 14 December 2007)

\begin{abstract}
The magnetization dynamics in magnetic double layers is affected by spin-pump and spin-sink effects. So far, only the spin pumping and its effect on the magnetic damping has been studied. However, due to conservation of angular momentum this spin current also leads to magnetic excitation of the layer dissipating this angular momentum. In this Letter we use time resolved magneto-optic Kerr effect to directly show the excitation due to the pure spin current. In particular, we observe magnetization dynamics due to transfer of angular momentum in magnetic double layers. In contrast to other experiments where a spin polarized charge current is passed through a nanomagnet, the effects discussed in this Letter are based on pure spin currents without net transfer of electric charge.
\end{abstract}

Spin polarized currents offer the possibility to exert a large torque on nanomagnets $[1,2]$. In recent years current induced magnetization dynamics was successfully demonstrated in columnar magnetic nanostructures [3-7] and ferromagnetic nanowires where the spin polarized current is used to manipulate magnetic domain walls $[8,9]$. Switching of a magnetic nanoparticle by a spin current was even demonstrated [10] in a nonlocal crossed wire geometry. In all these experiments the spin polarized currents are driven by charge currents. The large current densities needed to exert a sizable torque on the magnetization limit the size of the structures to a few hundred nanometers. In larger structures the classical magnetic field due to the charge current dominates the spin torque. Tserkovnyak et al. [11] have shown that magnetization dynamics itself can create pure spin currents without a net flow of electric charge at normal metal/ferromagnet interfaces. Evidence for this effect was found in the ferromagnetic resonance (FMR) line broadening observed in magnetic single [12] and double layers [13,14]. In magnetic single layers the spin accumulation due to spin pumping was also electrically detected [15]. In the ferromagnet/ normal metal/ferromagnet $(F 1 / N / F 2)$ double layer structures the second ferromagnet acts as a spin sink for the spin current pumped by the first ferromagnet [14]. The mutual exchange of spin currents between $F 1$ and $F 2$ leads to dynamic exchange coupling $[14,16]$. In contrast to static interlayer exchange coupling this coupling does not oscillate with the spacer thickness, it is almost independent of interface roughness, and its range is limited by the spin diffusion length [14]. So far, all evidence of this coupling is based on the broadening of FMR lines [13]. In the case of standard FMR the sum of the rf susceptibilities from both magnetic layers is measured and the only observable evidence of the dynamic coupling is an increase of the effective Gilbert damping $[13,14]$. This additional damping is a consequence of the loss of angular momentum due to spinpump and spin-sink effects.
For small precessional angles the magnetization dynamics in the presence of dynamic exchange coupling can be described by the following coupled set of Landau-LifshitzGilbert equations [14]:

$$
\begin{aligned}
\frac{d \vec{m}_{i}}{d t}= & -\mu_{0} \gamma\left[\vec{m}_{i} \times \vec{H}_{\text {eff }}\right]+\alpha_{i}\left[\vec{m}_{i} \times \frac{d \vec{m}_{i}}{d t}\right] \\
& +\alpha_{i}^{\mathrm{SP}}\left[\vec{m}_{i} \times \frac{d \vec{m}_{i}}{d t}-\vec{m}_{j} \times \frac{d \vec{m}_{j}}{d t}\right],
\end{aligned}
$$

where $\vec{m}_{1,2}$ are unit vectors along the instantaneous magnetization directions in $F 1$ and $F 2$. The strength of the spin-pump and spin-sink effects is given by the parameter $\alpha_{1}^{\mathrm{SP}}=g \mu_{B} \frac{g^{\Uparrow \downarrow}}{\mu_{0} M_{s}} \frac{1}{t_{1}}$, where $t_{1}$ is the thickness of $F 1, g^{\Uparrow \downarrow}$ (in units of $\left.e^{2} / h\right)$ is the real part of the spin mixing conductance $[14,17]$, and $\mu_{B}$ is the Bohr magneton. The exchange of spin currents is a symmetric concept and the equation of motion for layer $F 2$ can be obtained by interchanging the indices $i \rightleftarrows j$ in Eq. (1). The third and fourth terms on the right-hand side of Eq. (1) represent the spin-pump and spin-sink effects on the magnetization in $F 1$. The fourth term is generated by the precession (spin pump) of the magnetization in $F 2$. It is worthwhile to point out that the signs $(+)$ and $(-)$ in the third and fourth terms in Eq. (1) represent the spin current directions $(F 1 \rightarrow F 2)$ and $(F 2 \rightarrow$ $F 1$ ), respectively. Both layers, $F 1$ and $F 2$, act as mutual spin pumps and spin sinks. The $1 / t_{i}$ dependence of $\alpha_{i}^{\mathrm{SP}}$ shows that the dynamic exchange coupling is an interface effect.

The net spin current generated by spin pumping of layer $F 1$ propagates away from the $F 1 / N$ interface [11] and is absorbed at the $N / F 2$ interface if $N$ is thinner than the spin diffusion length (spin-sink effect) [18]. Conservation of angular momentum requires that layer $F 1$ will loose spin momentum which leads to an increase of the Gilbert damping parameter of layer $F 1$. Subsequently, the spin current absorbed at the $N / F 2$ interface creates an additional $\mathrm{rf}$ torque on the magnetic moment in $F 2$. Since $F 2$ is an 
ultrathin ferromagnet the interface torque results in a homogeneous precession of the layer $F 2$ at the resonance frequency of $F 1$. In standard FMR experiments this effect cannot be observed directly since the rf response is always dominated by the FMR signal from $F 1$. The purpose of this Letter is to demonstrate explicitly the rf excitation of layer $F 2$ (acting as a spin sink) by the spin current created at the $F 1 / N$ interface.

For the measurements, we employ time $(\sim 1 \mathrm{ps})$ and spatially $(\sim 300 \mathrm{~nm})$ resolved magneto-optical Kerr effect (TRMOKE) combined with continuous wave (cw) rf driving. Instead of applying a short magnetic field pulse to the magnetic system the sample is excited by means of a cw rf field which is created by a comb generator from high harmonics of the laser repetition rate and in doing so is inherently phase locked to the laser probe pulses. A single frequency from the comb is picked using a yttrium iron garnet filter and subsequently amplified. By recording the TRMOKE signal as a function of the applied magnetic field with a fixed microwave phase one can directly measure the real and imaginary parts of the rf susceptibility. The imaginary part of the susceptibility is measured by adjusting the microwave phase such that the TRMOKE signal is sensitive to the out-of-phase rf susceptibility. The real part of the rf susceptibility is detected in a second measurement by shifting the microwave phase by $\pi / 4$. In contrast to experiments with a pulsed excitation, which always leads to excitation of both magnetic layers in a double layer structure, the microwave excitation allows one to selectively excite dominantly one layer of the double layer at a given field.

The magnetic double layer structures were grown by molecular beam epitaxy in ultrahigh vacuum on $\mathrm{GaAs}(001)$ substrates [13]. In $\mathrm{Au} / \mathrm{Fe} / \mathrm{Au} / \mathrm{Fe} / \mathrm{GaAs}(001)$ structures the interface anisotropies of the two ferromagnetic layers can be used to split the resonance fields of $F 1$ and $F 2$ by $50 \mathrm{mT}$ at $10 \mathrm{GHz}$ [see Fig. 1(a)]. The results presented in this Letter are obtained using samples with the following structure: $20 \mathrm{Au} / 10 \mathrm{Fe} /$ spacer $/ 16 \mathrm{Fe} / \mathrm{GaAs}$, (the integers are in monolayers). The studies were carried out using $200 \mathrm{Au}(40 \mathrm{~nm})$ and $350 \mathrm{Ag}(70 \mathrm{~nm})$ normal metal spacers. The magnetic parameters of the two layers were determined by FMR and for the sample with the 200Au spacer given by $16 \mathrm{Fe}(\mathbf{F 1})$ : The perpendicular demagnetizing field $\mu_{0} M_{\text {eff }}=1.65 \mathrm{~T}, K_{U}=6.0 \times 10^{4} \mathrm{~J} / \mathrm{m}^{3}$, and $K_{1}=3.0 \times 10^{4} \mathrm{~J} / \mathrm{m}^{3}$ (hard axis parallel to [110]); 10Fe(F2): $\mu_{0} M_{\text {eff }}=1.25 \mathrm{~T}, K_{U}=0.3 \times 10^{4} \mathrm{~J} / \mathrm{m}^{3}$, and $K_{1}=2.0 \times 10^{4} \mathrm{~J} / \mathrm{m}^{3}$ (easy axis along $\langle 100\rangle$ directions). $K_{U}$ and $K_{1}$ are the in-plane uniaxial and fourfold anisotropies, respectively. The sample with the $350 \mathrm{Ag}$ spacer has very similar properties. Ag causes a larger perpendicular interface anisotropy compared to Au which reduces $M_{\text {eff }}$ of $F 1$ and $F 2$ by a few percent and leads to slightly higher resonance fields. The expected resonance frequencies for $F 1$ and $F 2$ as a function of bias field applied along the [110] direction are shown in Fig. 1(a). The $40 \mathrm{~nm}$ thick Au spacer layer was used in order to substantially decrease the

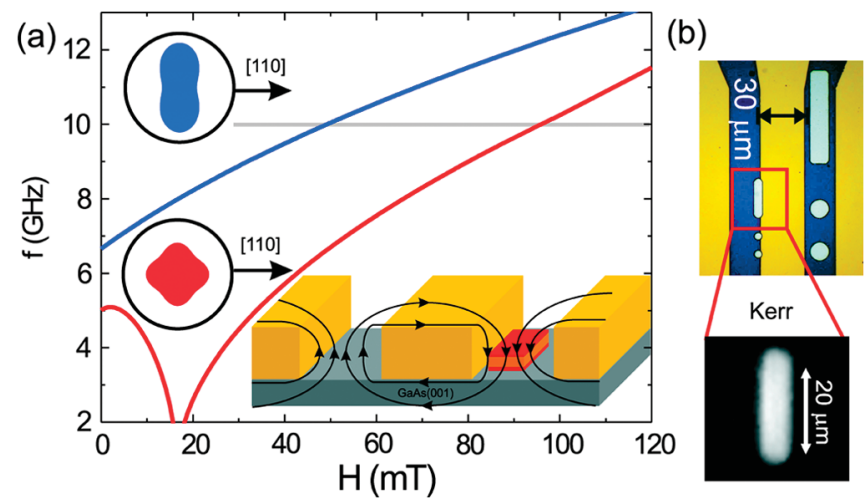

FIG. 1 (color). (a) Calculated resonance frequency versus applied field for the $20 \mathrm{Au} / 10 \mathrm{Fe} / 200 \mathrm{Au} / 16 \mathrm{Fe} / \mathrm{GaAs}(001)$ sample with the magnetic field applied parallel to the [110] direction of $\mathrm{Fe}$. The FMR frequencies for the $16 \mathrm{Fe}(F 1)$ and $10 \mathrm{Fe}(F 2)$ layers are shown in the blue line $(F 1)$ and red line $(F 2)$, respectively. The difference between the red line and the blue line is caused by the in-plane uniaxial interface anisotropy at the $F 1 / \mathrm{GaAs}$ interface with the easy axis along the [110] direction. Layer $F 2$ has a weak cubic anisotropy with the easy magnetic axes along $\langle 100\rangle$. Polar plots of the effective magnetic anisotropy energy are shown in blue $(F 1)$ and red $(F 2)$, respectively. The circle around the polar plot corresponds to an energy of $10^{5} \mathrm{~J} / \mathrm{m}^{3}$. The horizontal gray line at $10 \mathrm{GHz}$ indicates the expected resonance fields at this frequency. The inset shows a schematic view of a coplanar waveguide structure. The rf-driving field is out of plane and $F 1$ and $F 2$ are driven in phase. (b) Optical micrograph of the actual sample. The signal line has a width of $30 \mu \mathrm{m}$. The magnification shows the Kerr signal of the $25 \times 8 \mu \mathrm{m}^{2}$ rectangle in a bias field of $65 \mathrm{mT}$ at a frequency of $8.08 \mathrm{GHz}$ (resonance of F2).

Kerr signal from the bottom layer $F 1$. Hamrle et al. have shown that for a $40 \mathrm{~nm}$ thick Au cap layer the polar Kerr effect is suppressed by about $85 \%$ at a wavelength of $400 \mathrm{~nm}$ [19].

The configuration we use for the TRMOKE measurements is shown in the inset of Fig. 1(a). A small island of the epitaxial structure is defined by optical lithography and dry etching. Subsequently, a $200 \mathrm{~nm}$ thick Au coplanar waveguide is prepared by optical lithography, thermal evaporation, and lift-off processes. The epitaxial layer structure is located in the gap of the coplanar waveguide between the signal conductor and the ground conductors [cf. Fig. 1(a)]. For this configuration the rf-magnetic field is out of plane and in phase in both layers $F 1$ and $F 2$. The waveguide is oriented parallel to the [110] direction of the Fe films and the magnetic dc field is applied parallel to the waveguide. In Fig. 1(b) we show an optical micrograph and the Kerr signal at FMR across the $25 \times 8 \mu \mathrm{m}^{2}$ epitaxial island. From the uniformity of the magnetic response it is evident that dipolar effects are very weak for these thin layers. The residual Kerr signal from the bottom layer can be entirely suppressed by the depth selectivity of the Kerr effect. In a Kerr microscope this is achieved by using a rotatable compensator (e.g., 1/4 wave plate). In doing so, 
the phase of the Kerr signal can be adjusted relative to the reflected light. In this way, the Kerr signal from a selected depth region can be made invisible. This technique was first demonstrated by Hubert and Schäfer for $\mathrm{Fe} / \mathrm{Cr} / \mathrm{Fe}$ multilayers [20].

Figure 2(a) shows the in- and out-of-phase parts of the magnetic response of $F 2$ measured by TRMOKE. A cw signal at $10 \mathrm{GHz}$ was applied to the coplanar transmission line and as expected from Fig. 1(a) the main FMR signal for the layer $F 2$ occurs at a field of $95 \mathrm{mT}$. At the resonance field of layer $F 1$ a typical "dispersion wiggle" (corre-
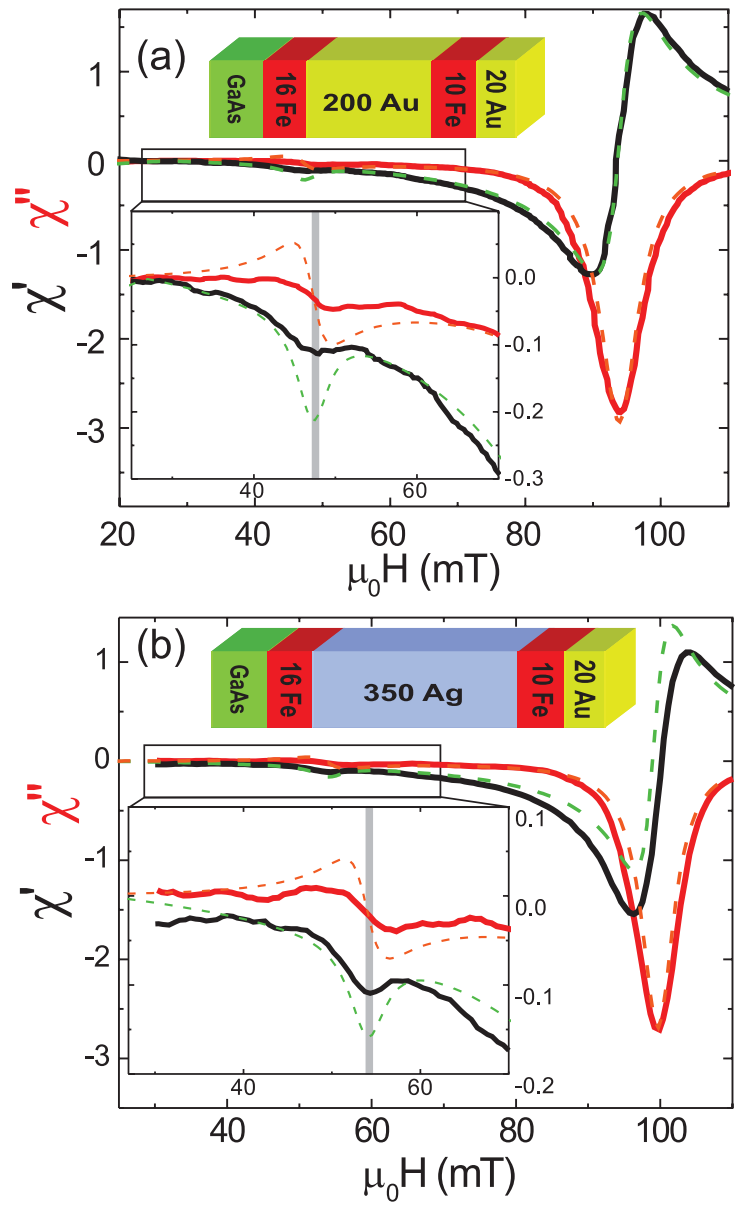

FIG. 2 (color). (a) Sample 20Au/10Fe/200Au/16Fe/ $\mathrm{GaAs}(001)$. Experimentally measured real (red line) and imaginary (black line) parts of the perpendicular rf susceptibility of layer $F 2$. A rf frequency of $10 \mathrm{GHz}$ was used and the magnetic dc field was swept parallel to the [110] direction. The corresponding calculations using Eq. (1) are shown as dotted lines. The inset magnifies the region of interest. In the calculation the following spin-pump, spin-sink, and damping parameters were used: $\alpha_{F 1}=\alpha_{F 2}=0.0045, \alpha_{F 1}^{\mathrm{SP}}=0.0030$, and $\alpha_{F 2}^{\mathrm{SP}}=0.0050$. The fact that the thinner layer has stronger $\alpha^{\mathrm{SP}}$ is due to the $1 / t$ dependence of the effect and consistent with the FMR results reported in [13]. (b) Sample $20 \mathrm{Au} / 10 \mathrm{Fe} / 350 \mathrm{Ag} / 16 \mathrm{Fe} /$ $\operatorname{GaAs}(001)$. The experimental data are shown as red line $\left(\chi^{\prime \prime}\right)$ and black line $\left(\chi^{\prime}\right)$. Dotted lines are the corresponding calculation using the following parameters: $\alpha_{F 1}=\alpha_{F 2}=0.0040$, $\alpha_{F 1}^{\mathrm{SP}}=0.0030$ and $\alpha_{F 2}^{\mathrm{SP}}=0.0050$. sponding to the real part of rf susceptibility $\chi^{\prime}$ ) and a "dip" (corresponding to the imaginary part of the rf susceptibility $\chi^{\prime \prime}$ ) are found in the tails of the out-phase and inphase rf response, respectively. These signals at the $F 1$ resonance field are in agreement with the spin-pumping theory. $F 2$ is driven by the spin current generated by $F 1$, see Eq. (1), which is proportional to the time derivative of the $\mathrm{rf}$ magnetization of layer $F 1$. Therefore this driving is phase shifted by $\pi / 2$ with respect to the $\mathrm{rf}$ magnetization. Consequently, the additional driving due to spin pumping exchanges the resonance line shapes for the real and imaginary parts in the $\mathrm{rf}$ susceptibility. That is, in the imaginary (real) part of the $F 2$ susceptibility this results in a contribution at the $F 1$ resonance which looks like the real (imaginary) part of the susceptibility of $F 1$; see Figs. 2(a) and 2(b). All these experimental features are well reproduced by the spin-pumping theory using Eq. (1). The measured signal agrees with the calculations in its shape; however, because of spin scattering the amplitude is reduced by $70 \%$ compared to ballistic spin transport [14] [cf. dashed lines in Figs. 2(a) and 2(b)].

One cannot a priori exclude other contributions to the signal observed at the field where the spin-pumping effect due to the precession of layer $F 1$ is observed. They are as follows: (i) a residual TRMOKE signal from the layer $F 1$ and (ii) dipolar and exchange coupling fields between the two layers. (i) is addressed by using a rotatable compensator: Fig. 3(a) shows clearly that by rotation of the optical compensator $(\lambda / 4$ plate $)$ the Kerr signal originating from the bottom layer can be entirely suppressed. It is important to note that the direct signal from layer $F 1$ has as expected the field dependence corresponding to that of the main FMR signal; see Fig. 3(a). In Fig. 3(a) the spin pumping leads to a "dispersion wiggle" (odd function in field) while the direct FMR signal leads to a dip (even function in field).

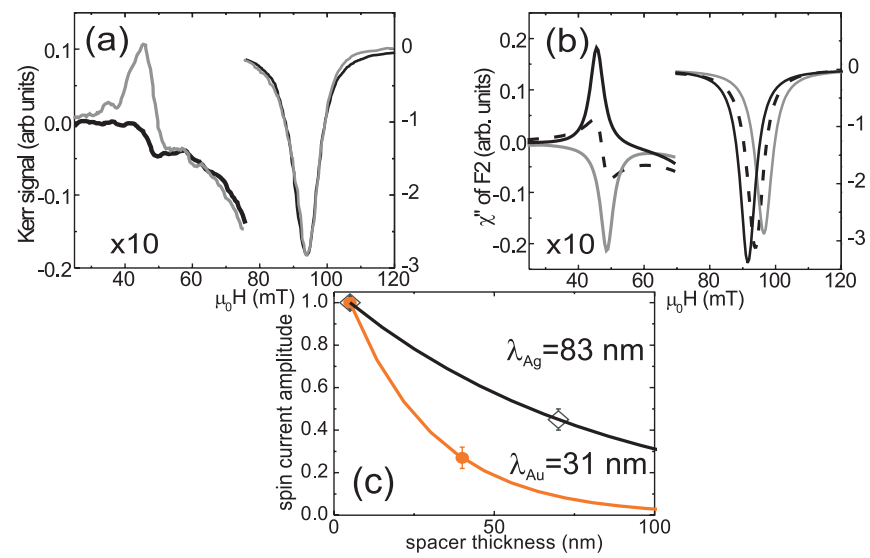

FIG. 3 (color online). (a) Measurement of $\chi^{\prime \prime}$ : Suppression of the spurious signal (gray line) from $F 1$ using the $1 / 4$ wave plate (black line). (b) Simulation of $\chi^{\prime \prime}$ of $F 2$ for weak ferromagnetic (gray line) and antiferromagnetic (black line) coupling. The dashed line is the expected signal due to spin pumping. (c) Estimation of the spin diffusion length from the data shown in Fig. 2. 
Therefore one can easily distinguish between the signals caused by spin pumping and the direct Kerr signal from layer $F 1$ by inspecting the field dependence of the resonance measured at the FMR field of $F 1$. This leads to a unique situation: By using depth resolved Kerr effect one can suppress entirely the Kerr signal from the bottom layer $F 1$ but remains sensitive to the top layer $F 2$. This perfect cancellation is easy to achieve because our films are much thinner than the optical wavelength inside the metal. Because of the shorter optical attenuation length and a higher reflectivity at $400 \mathrm{~nm}$ of $\mathrm{Ag}$ [21] no direct Kerr signal was observed in the sample with the $350 \mathrm{Ag}$ spacer and the above procedure was not required. (ii) The exchange coupling and dipolar fields are proportional to the rf-magnetization components. This means they would again result in a resonance signal at the FMR field of $F 1$ similar to the direct Kerr signal from layer $F 1$. In addition the coupling fields are very weak. The direct exchange coupling can be entirely excluded because our Au spacer is too thick. The short wavelength oscillatory coupling is effectively averaged out by interface roughness for the $\mathrm{Au}$ spacers thicker than $3 \mathrm{~nm}$. The strength of dipolar coupling can be estimated in the following way: The dipolar field in the ultrathin film with the inhomogeneous magnetization caused by the finite size of the sample is given by $\mu_{0} H_{D} \sim$ $\frac{1}{2} q t_{F} \mu_{0} M_{S} \sin \phi$, where $q$ is the wave number of the inhomogeneous rf magnetization, $t_{F}$ is the thickness of the ferromagnet, and $\phi$ is the cone angle of the rf precession. The $q$ wave number is given by $q=\pi / \lambda$, where $\lambda$ is an appropriate wavelength of the inhomogeneous rf magnetization given by the sample's lateral geometry. Using conservative numbers to estimate the field $\phi=0.5^{\circ}$, $t_{F}=2 \mathrm{~nm}, t_{F}=40 \mathrm{~nm}, \lambda=5 \mu \mathrm{m}$ results in $H_{D}=1 \times$ $10^{-4} \mathrm{mT}$. Considering that the excitation field is about $0.5 \mathrm{mT}$ it is obvious that the driving due to dipolar coupling is at least an order of magnitude weaker than the driving by spin pumping. In addition, dipolar coupling would always lead to a peak or dip at the $F 1$ resonance but never result in the observed "dispersion wiggle" [cf. Fig. 3(b)]. Therefore one can firmly conclude that the signal measured at the $F 1$ resonance in Figs. 2(a) and 2(b) is a sole consequence of the absorbed spin current in $F 2$.

In addition, we would like to emphasize that the technique presented here can be used to estimate the spin diffusion length. $\mathrm{Ag}$ is a lighter element than $\mathrm{Au}$ which results in a smaller spin orbit interaction and a longer spin diffusion length. The data shown in Fig. 2(b) indicate that although the $\mathrm{Ag}$ spacer is almost twice as thick as the $\mathrm{Au}$ spacer the signal due to spin pumping is approximately 2 times larger compared to the signal measured with a 200Au spacer [cf. Figs. 2(a) and 2(b)]. Only $53 \pm 5 \%$ of the spin current is lost in the Ag spacer. The maximum effect from spin pumping for very thin spacer layers was determined by FMR experiments [14] and corresponds to ballistic spin transport across $N$. This value and the measurements for the $200 \mathrm{Au}$ and $350 \mathrm{Ag}$ spacer allow one to estimate the spin diffusion lengths in $\mathrm{Au}$ and $\mathrm{Ag}$; see Fig. 3(c). From a simple exponential fit one obtains $\lambda_{\mathrm{Au}}=$ $31 \pm 5 \mathrm{~nm}$ for $\mathrm{Au}$ and $\lambda_{\mathrm{Ag}}=83 \pm 10 \mathrm{~nm}$ for $\mathrm{Ag}$, respectively. These numbers are in fair agreement with results from transport measurements [22].

We have shown that the exchange of pure spin currents due to spin pumping leads to excitation of magnetization dynamics in $F 1 / N / F 2$ spin valve structures. The results obtained in field sweep measurements are in good agreement with the spin-pumping theory and clearly support the model of dynamic spin current exchange coupling in spin valves. The present data show that nonlocal spin dynamics due to pure spin currents is a sizable effect in magnetic multilayers. In addition, these experiments provide a new technique to study the spin diffusion length in normal metals spacers without the net transport of electric charge.

The authors would like to thank B. Kardas for assistance in the preparation of the samples. Financial support from the DFG Priority Programme 1133, DFG Sonderforschungsbereich 689, and Natural Sciences and Engineering Council of Canada is gratefully acknowledged.

[1] L. Berger, Phys. Rev. B 54, 9353 (1996).

[2] J. C. Slonczewski, J. Magn. Magn. Mater. 159, L1 (1996).

[3] M. Tsoi et al., Phys. Rev. Lett. 80, 4281 (1998).

[4] J. A. Katine, F. J. Albert, R. A. Buhrman, E. B. Myers, and D. C. Ralph, Phys. Rev. Lett. 84, 3149 (2000).

[5] S. I. Kiselev et al., Nature (London) 425, 380 (2003).

[6] W.H. Rippard, M. R. Pufall, S. Kaka, S. E. Russek, and T. J. Silva, Phys. Rev. Lett. 92, 027201 (2004).

[7] I. N. Krivorotov et al., Science 307, 228 (2005).

[8] M. Klaui et al., Phys. Rev. Lett. 95, 026601 (2005).

[9] M. Hayashi et al., Nature Phys. 3, 21 (2007).

[10] T. Kimura, Y. Otani, and J. Hamrle, Phys. Rev. Lett. 96, 037201 (2006).

[11] Y. Tserkovnyak, A. Brataas, and G.E.W. Bauer, Phys. Rev. Lett. 88, 117601 (2002).

[12] S. Mizukami, Y. Ando, and T. Miyazaki, J. Magn. Magn. Mater. 226-230, 1640 (2001).

[13] R. Urban, G. Woltersdorf, and B. Heinrich, Phys. Rev. Lett. 87, 217204 (2001).

[14] B. Heinrich et al., Phys. Rev. Lett. 90, 187601 (2003).

[15] M. Costache et al., Phys. Rev. Lett. 97, 216603 (2006).

[16] E. Šimánek and B. Heinrich, Phys. Rev. B 67, 144418 (2003).

[17] A. Brataas, Y. Tserkovnyak, G.E.W. Bauer, and B.I. Halperin, Phys. Rev. B 66, 060404(R) (2002).

[18] M.D. Stiles and A. Zangwill, Phys. Rev. B 66, 014407 (2002).

[19] J. Hamrle, J. Ferré, M. Nývlt, and Š. Višňovský, Phys. Rev. B 66, 224423 (2002).

[20] A. Hubert and R. Schäfer, Magnetic Domains. The Analysis of Magnetic Microstructures (Springer, New York, 2000).

[21] J. Hohlfeld et al., Appl. Phys. B 64, 387 (1997).

[22] R. Godfrey and M. Johnson, Phys. Rev. Lett. 96, 136601 (2006). 